\title{
Correction: Li, S. et al. Research on a Power Management System for Thermoelectric Generators to Drive Wireless Sensors on a Spindle Unit. Sensors 2014, 14, 12701-12714
}

\section{Sheng Li ${ }^{1,2, *}$, Xinhua Yao ${ }^{1}$ and Jianzhong Fu ${ }^{1}$}

1 The State Key Laboratory of Fluid Power Transmission and Control, Zhejiang University, Hangzhou 310027, China; E-Mails: yaoxinhuazju@gmail.com (X.Y.); fjz@zju.edu.cn (J.F.)

2 School of Mechanical Engineering, Hangzhou Dianzi University, Hangzhou 310018, China

* Author to whom correspondence should be addressed; E-Mail: ttaidttaid@163.com;

Tel./Fax: +86-571-8691-9054.

Received: 7 August 2015 / Accepted: 11 August 2015 / Published: 20 August 2015

The authors wish to make the following corrections to this paper [1]. The authorship should be "Sheng $\mathrm{Li}^{1,2, *}$, Xinhua Yao ${ }^{1}$ and Jianzhong $\mathrm{Fu}{ }^{1}$ ", and the affiliations should be changed from:

1 School of Mechanical Engineering, Hangzhou Dianzi University, Hangzhou 310018, China

2 The State Key Lab of Fluid Power Transmission and Control, Zhejiang University, Hangzhou 310027, China; E-Mails: yaoxinhuazju@gmail.com (X.Y.); fjz@zju.edu.cn (J.F.) to:

1 The State Key Laboratory of Fluid Power Transmission and Control, Zhejiang University, Hangzhou 310027, China; E-Mails: yaoxinhuazju@gmail.com (X.Y.); fjz@zju.edu.cn (J.F.)

2 School of Mechanical Engineering, Hangzhou Dianzi University, Hangzhou 310018, China

Also, the Acknowledgments section should be changed from:

"This work is supported by the National Natural Science Foundation of China (No. 51105336) and the National Natural Science Foundation of China (No. 61100101)."

to:

"This work is supported by the National Natural Science Foundation of China (No. 51105336), the National Natural Science Foundation of China (No. 61100101) and the Fundamental Research Funds for the Central Universities."

The authors would like to apologize for any inconvenience caused to the readers by these changes. 


\section{Conflicts of Interest}

The authors declare no conflicts of interest.

\section{References}

1. Li, S.; Yao, X.; Fu, J. Research on a Power Management System for Thermoelectric Generators to Drive Wireless Sensors on a Spindle Unit. Sensors 2014, 14, 12701-12714.

(C) 2015 by the authors; licensee MDPI, Basel, Switzerland. This article is an open access article distributed under the terms and conditions of the Creative Commons Attribution license (http://creativecommons.org/licenses/by/4.0/). 